\title{
The improvement on Respiratory Muscle, Trunk Stability, and health status after Abdominal Drawing-in Maneuver in COPD Patients
}

\author{
Winny $^{1}$, Siti Chandra Widjanantie ${ }^{2}$, Maryastuti ${ }^{3}$, Nury Nusdwinuringtyas ${ }^{4}$
}

Resident of Physical Medicine and Rehabilitation, Faculty of Medicine University of Indonesia Department of Physical Medicine and Rehabilitation, Persahabatan General Hospital Department of Radiology, Persahabatan General Hospital Department of Physical Medicine and Rehabilitation, Dr. Cipto Mangunkusumo National General Hospital

\begin{abstract}
Background: Chronic Obstructive Pulmonary Disease (COPD) patients experienced respiratory muscle dysfunction, postural instability, and decreasing in health status. Abdominal drawing-in maneuver (ADIM) has been studied in many cases of low back pain for lumbar stabilization, moreover this maneuver is also designed to activate the transversus abdominalis (TA) muscle that involved in expiration. But this exercise has not been considered as a respiration exercise in COPD patients. The purpose of this study was to determine whether the application of ADIM to COPD patients would affect the strength of respiratory muscle, improve core muscle stability, and health status of COPD patients.

Methods:All clinically stable COPD patients who visited PMR clinic at Persahabatan General Hospital were recruited in the study. They received exercise interventions 2 times a week for 4 weeks. ADIM as a main program is using pressure transducer (Chattanooga, Australia). Each exercise was held 10 repetitions, 10 sets with 2 minutes rest. Strength of respiratory muscle measured by peak cough flow (PCF) and peak flow rate (PFR). Core muscle stability measured by functional reach test (FRT) and the health status measured with CAT score. The measurements were done before and immediately after intervention.

Results: Subjects were 8 patients with mean age 62 years old, consisted of 7 men and 1 woman, with 1 patient each with COPD grade A, B, and C, and 5 patients with COPD grade D. There were increasing of PCF (268.75 $\pm 59.146 \mathrm{~L} / \mathrm{min}$ to $285.00 \pm 59.522 \mathrm{~L} / \mathrm{min} ; \mathrm{p}=0.061)$, PFR $(251.3 \pm 96.3 \mathrm{~L} / \mathrm{min}$ to $286.3 \pm 92 \mathrm{~L} / \mathrm{min} ; \mathrm{p}=0.028)$, FRT $(20.2 \pm 3.8 \mathrm{~cm}$ to $22 \pm 3.9 \mathrm{~cm} ; \mathrm{p}=0.011)$, and decreasing of CAT score $(14 \pm 8.685$ to $11.50 \pm 8.848 ; \mathrm{p}=0.027)$ after ADIM.
\end{abstract}

Conclusion: There were an improvement in respiratory muscle, trunk stability, and CAT after ADIM, so the maneuver is effective for COPD management.

Keywords: Abdominal drawing-in maneuver (ADIM), Chronic Obstructive Pulmonary Disease (COPD), COPD Assessment Test (CAT), Functional reach test (FRT), Peak cough flow (PCF), Peak flow rate (PFR) 


\section{Correspondence Detail:}

Wini

Email: winnywinaldy10@gmail.com

Department of Physical Medicine and Rehabilitation,

Medical Faculty of University of Indonesia, Dr. Cipto Mangunkusumo National General Hospital Jakarta, Indonesia

\section{INTRODUCTION}

Chronic Obstructive Pulmonary Disease (COPD) is a common, preventable and treatable disease that is characterized by persistent respiratory symptoms and airflow limitation that is due to airway and/or alveolar abnormalities usually caused by significant exposure to noxious particles or gases. The most common respiratory symptoms include dyspnea, cough and/or sputum production. Since medications do not relieve all COPD symptoms nor cure the illness, pulmonary rehabilitation should be consider as a strategy to improve dyspnea, cough andspu tum production. ${ }^{1}$

There was respiratory muscle dysfunction in COPD, due to cytokine and muscle wasting. Respiratory muscle dysfunctions have significant consequences for COPD patients. ${ }^{2}$ Expiratory (abdominal muscles, internal intercostal) and inspiratory (diaphragm, external intercostal) muscle strength can be impaired in patients with COPD and significantly affects the quality of life of patients. ${ }^{3}$

The consequence of inspiratory muscle weakness is the loss of ability to perform a deep inspiration, which is necessary to maintain peripheral alveolar ventilation in addition to affecting the first phase of cough. The weakness of the expiratory muscles may be seen in a reduction in maximal expiratory pressure and reduced ability to perform a deep expiration and cough. The peak cough flow (PCF) as a functional marker in COPD would therefore appear to address both inspiratory and expiratory muscle strength with an advantage of giving us an idea about cough strength. While the peak flow rate (PFR) reflects large airway flow and depends on the voluntary effort and muscular strength of the patient. ${ }^{3}$

In addition, diaphragm and transversus abdominalis (TA) also is one of the muscles that keep the core muscle stability. Optimal stabilization of core muscle, may provide adequate postural control. This becomes important in COPD patients because most of COPD patients are elderly so they are high risk of falling. Therefore, by maintaining the stability of the core is expected to improve the function of patient's balance. Functional reach test (FRT) is one of the reliable measurements of balance that may be useful in the clinical assessment of instability. Reaching is a ubiquitous function that constantly imposes a stress to patient's balance. ${ }^{4}$

The abdominal drawing-in maneuver (ADIM) is commonly used in lumbar stabilization training programs, however the maneuver is designed to activate the TA muscle as well. The abdominal muscles, including the TA, are also the most powerful muscles involved in expiration. Expiratory muscle can be specifically as a targeted training in pulmonary rehabilitation programs with improvement of both strength and endurance in patients with COPD. The 
ADIM can possibly increase expiratory muscle strength due to transversus abdominis muscle activation. $^{5}$

While the ADIM has been studied in many cases of low back pain, this exercise has not been considered as a respiration exercise in COPD patients. Therefore, the purpose of this study was to determine whether the application of ADIM to COPD patients would affect respiratory muscle especially diaphragm, trunk stability and COPD assessment test (CAT).

\section{METHODS}

The participants in this study were all clinically stable COPD patients who visited Rehabilitation Medicine outpatient clinic at Persahabatan General Hospital from May to June 2018. Each subject received exercise interventions 2 times a week for 4 weeks. Stretching and breathing exercise were carried out as a warm up and cooling down, while ADIM as a main program using pressure transducer (Chattanooga, Australia). Each exercise was held 10 repetitions and 10 sets. The pressure transducer was placed centrally, behind the lumbar spine, from S2 to approximately L1. Baseline levels in the pressure biofeedback were standardized to $4 \mathrm{O} \mathrm{mmHg}$. After that, the subject were trained to expand the lateral lower ribcage in a lateral direction during the inspiratory phase of tidal breathing and minimal superior movements of the chest then the lower abdomen was expanded. After that, pull their abdomen toward spine until the pressure gauge, which is connected to the pressure biofeedback unit, to incline up around 2-4 mmHg. Subjects were then taught to preferentially activate their
TA muscle by performing the ADIM with visual feedback from the pressure biofeedback unit (PBU). The tests were based on peak cough flow (PCF), peak flow rate (PFR), functional reach test (FRT) and COPD assessment test (CAT) measured immediately before and after intervention.
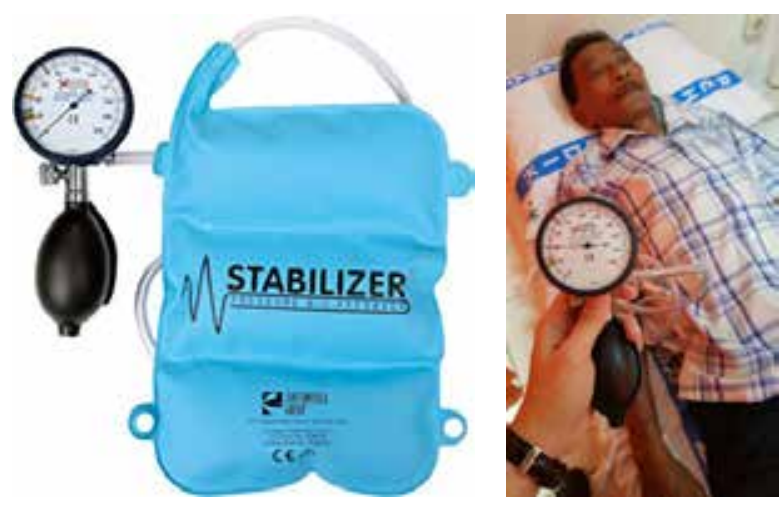

Figure 1. A. Pressure transducer (Chattanooga, Australia) as a pressure biofeedback unit (PBU); B. ADIM exercise using pressure transducer

The data were managed by SPSS 20 program. The paired T-test or Wilcoxon test used to evaluate the differences between PCF, PFR, FRT and CAT score test before and after intervention.

\section{RESULTS}

The nine clinically stable of COPD patients recruited, then one of patient has incomplete the intervention. The mean age was 62.00 years old, consisted of 7 men and 1 woman, with CAT Score was 14.0 \pm 8.69 . Most of patients $(62.5 \%)$ had grade $\mathrm{D}$ of COPD, and the rest were one subject in grade A, B, and C. All of subjects had medical treatment for concomitant diseases. The characteristics of the patients were shown in Table 1. 
Table 1. Characteristics of Subjects

\begin{tabular}{ll}
\hline & n (percentage) \\
\hline Age (mean \pm SD) & $62.00 \pm 7.75$ \\
Gender & $7(87.5 \%)$ \\
Man & $1(12.5 \%)$ \\
Woman & $14.0 \pm 8.69$ \\
CAT SCORE (mean \pm SD) & \\
COPD Grade & $1(12.5 \%)$ \\
A & $1(12.5 \%)$ \\
$C$ & $1(12.5 \%)$ \\
$D$ & $5(62.5 \%)$ \\
\hline
\end{tabular}

COPD $=$ Chronic Obstructive Pulmonary Disease; CAT Score $=$ COPD Assessment Test

Table 2 showed there were tend to increase of PCF before and after ADIM $(268.75 \pm 59.146 \mathrm{~L} / \mathrm{min}$ to $285.00 \pm 59.522 \mathrm{~L} / \mathrm{min} ; \mathrm{p}=0.061)$. While, there was significant increasing of PFR $(251.3 \pm 96.3$
$\mathrm{L} / \mathrm{min}$ to $286.3 \pm 92 \mathrm{~L} / \mathrm{min}$; with $\mathrm{p}=0.028)$, FRT $(20.2 \pm 3.8 \mathrm{~cm}$ to $22 \pm 3.9 \mathrm{~cm} ; \mathrm{p}=0.011)$, and decreasing of CAT score (14 \pm 8.685 to 11.50 \pm 8.848 ; $\mathrm{p}=0.027$ ) after ADIM exercise.

Table 2. Pre and Post ADIM Comparison of PCF, PFR, and FRT

\begin{tabular}{lccc}
\hline \multicolumn{1}{c}{ Variable } & Pre & Post & P value \\
\hline PCF & $268.75 \pm 59.146$ & $285.00 \pm 59.522$ & .061 \\
PFR & $251.25 \pm 96.279$ & $286.25 \pm 91.953$ & .028 \\
FRT & $20.200 \pm 3.8165$ & $22.838 \pm 3.9471$ & .011 \\
CAT Score & $14 \pm 8.685$ & $11.50 \pm 8.848$ & .027 \\
\hline
\end{tabular}

Wilcoxon test

$\mathrm{PCF}=$ peak cough flow; $\mathrm{PFR}=$ peak flow rate; $\mathrm{FRT}=$ functional reach test CAT score $=\mathrm{COPD}$ assessment test.

\section{DISCUSSION}

7 men and 1 woman stable COPD patients under treatment mean age over 60 years old, with different degree of COPD were recruited in this study. Stable patients and under treatment were selected so they can follow the exercise well. The small number of samples and differences in COPD degree could make the results biased.

ADIM is a spinal stabilization exercise. Diaphragm, pelvic floor, and control intraabdominal pressure (IAP) and provide the stability of lumbopelvic posture. When the spinal stabilization exercise is applied, the 
method of pushing the abdomen out through abdominal breathing is used. This method can do the co-contraction of the diaphragm and deep stability muscles and increase the intraabdominal pressure if the navel is maintained in a front-downward while the thoracic cage is not extended and the lower abdomen is expanded in the phase of inhalation. Another previous study also shows that the ADIM increases the abdominal deep muscle activity of Transversus Abdominis (TA). ${ }^{5}$ TA is the most powerful muscles involved in expiration. One of the expiratory muscle strength markers that can be seen is patient's coughing ability (PCF).

In this study, after four weeks application of ADIM, showed improvement in all five variables (PCF, PFR, FRT, and CAT score), but not significant improvement in PCF. Although it is not significant, there is still an increase in the PCF after ADIM exercise. It could be not significant because of the small number of sample size and the difference severity in COPD. The improvement of PCF were inline with previous study that showed deep abdominal muscle such as the TA and multifidus muscle contribute not only to stabilization of the spine, and adjustment of posture, but also to significant improvements in pulmonary function, when ADIM is applied. ${ }^{6}$

Peak expiratory flow measurement (peak flow) is a simple measure of the maximal flow rate that can be achieved during forceful expiration following full inspiration. It reflects large airway flow and depends on the voluntary effort and muscular strength of the patient. As mentioned above, ADIM could strengthen the TA, re-educates the respiratory muscles, improving muscle strength and endurance, as well as inducing powerful contraction of the respiratory muscles through repetitive afferent stimulation of the abdominal muscles. This increases intra-abdominal pressure, facilitating upward movement of the diaphragm, and decreases pleural pressure and lung volume, improving expiratory and sputum discharge abilities. So, after ADIM flow rate and health status of COPD patients will improve. It was proven in this study, that ADIM could improve significantly to the flow of the airway, marked by the improvement in PFR after the exercise, and decreasing the CAT score, which meant the health status increased. ${ }^{6}$

Previous studies stated COPD patients had postural instability that can increase the risk of falls. It can be caused of prolong hypoxia damage the audio-vestibular function, muscle weakness, elderly age, physical inactivity, and limited mobility. ${ }^{7,8}$ ADIM as already mentioned before, strengthen the respiration muscle especially TA, improve the flow of oxygen, so minimalized the hypoxia of audio-vestibular function. In addition, TA forms the walls of a cylinder while the muscles of the pelvic floor and diaphragm form its base and lid. This cylinder of compression influences intra-abdominal pressure (IAP) that is thought to contribute to spinal stability. ${ }^{9}$ This study has proven that ADIM increasing the strength of TA, improved the posture stability, and as the results were increased the PCF, PFR, FRS, as well as decreased of CAT.

\section{CONCLUSION}

There was an improvement in respiratory muscle, trunk stability, and health status on COPD patients. 


\section{REFERENCES}

1. Global initiative for chronic obstructive lung disease (GOLD). Pocket guide to COPD diagnosis, management, and prevention. 2017.

2. Otteenheijm et al. Diaphragm dysfunction in chronic obstructive pulmonary disease. Am J Respir Crit Care Med. 2005; 172: 200-205.

3. Sun Mi Kim, et al. A comparison of cough assistance techniques in patients with respiratory muscle weakness. Yonsei Medical Journal. 2016; 57(6):1488-1493.

4. Azis AE, Elwahsh RA, Abdelaal GA, Abdullah MS, Saad RA. Diaphragmatic assessment in COPD patients by different modalities. Egyptian journal of chest diseases and tuberculosis 66. 2017: 247-250.

5. Yoon MR, Choi HS, Shin WS. Effects of abdominal drawing-in maneuver and the abdominal expansion maneuver on grip strength, balance, and pulmonary function in stroke patients. Journal korea pt. 2015: 27(3):147-153.

6. Mantri B, Pattnaik M and Mohanty P. Effect of static abdominals training and incentive spirometer in improvement of pulmonary function and abdominal strength in spinal cord injury patients-A comparative study. Spine research. 2017;3(3):1-7.

7. Roig M, Eng JJ, Maclntyre DL, Road JD, and Reid WD. Postural control is impaired in people with COPD: an observational study. Physiother Can. 2011; 63(4):423-431.

8. Porto EF, Castro AAM, Schmidt VGS, Rabelo HM, Kumpel C, Nascimento OA, and Jardim JR. Postural control in chronic obstructive pulmonary disease: a systematic review. International Journal of Chronic Obstructive Pulmonary Disease. 2015; 10:1233-1239.

9. Nelson, Nicole MS, and LMT. Diaphragmatic breathing: The foundation of core stability. Strength and conditioning journal. 2012; 34(5):34-40. 